\title{
Computation of bond incident degree (BID) indices of complex structures in drugs
}

\author{
Jia-Bao Liua $^{\text {( }}$ |Abdul Qudair Baigb ${ }^{\circ}$ |Muhammad Imranc,* |Waqas Khalidb $^{\circ}$ |Muhammad $^{\circ}$ \\ Saeedd | Mohammad Reza Farahanie
}

${ }^{a}$ School of Mathematics and Physics, Anhui Jianzhu University, Hefei 230601, China

The molecular topological descriptors are the numerical invariants of a molecular graph and are very useful and efficient to predict their bioactivity. Dendrimers are highly branched organic macro-molecules with successive layers or generations of branch units surrounding a central core and have many applications in biology, medicine and drug designs. These are key molecules in nanotechnology and can be put to good use. A great variety of such indices are studied and used in theoretical chemistry and pharmaceutical researchers. Among them, a large number of indices depend only on vertex degrees of the molecular graph. A considerable amount of these vertex-degreebased topological indices can be represented as the sum of edge contributions of graph. These kind of vertex-degree-based topological indices are known as bond incident degree (BID) indices. In this paper, we determine the bond incident degree indices of complex structures in drugs called nanostar dendrimers and compute the closed formula for these indices.

*Corresponding Author:

Muhammad Imran

\section{KEYWORDS}

Email: m.imran658@uaeu.ac.ae

Tel.: +923334736997

Molecular structure descriptor; bond incident degree index; nanostar dendrimers; drug.

\section{Introduction}

A large number of new drugs have been manufactured each year with the increase of development in medicine manufacturing industry. Hence, there is a demand of tremendous amount of research work to determine the pharmacological, chemical and biological properties of these new developed drugs, and such workloads become more and more fussy and clustered. It requires enough reagents equipment and assistants to test the performances, effectiveness and the side effects of the new existing drugs. Nevertheless, in lower-income countries and underdeveloped areas (like certain cities and countries in South America, Africa and Southeast Asia), there is no sufficient money to defray reagents and equipment which can be employed to measure the biochemical properties of these new developed drugs. Fortunately, many previous studies have pointed that chemical and pharmacodynamics characteristics of drugs and their molecular structures are closely linked. The family of smart polymer is extensively used in anticancer drugs manufacturing [18]. The forgotten topological index is calculated of several widely used chemical structures which often appear in drug molecular graphs and various classes of drugs $[16,19]$. The extremal vertex-weighted Wiener number of bi-cyclic 
molecular graph in terms of molecular structural analysis and graph transformations was studied by Gao and Wang in [17]. A carbon nanotube network is motivated by the molecular structure of a regular hexagonal lattice and determined $\mathrm{R}_{\alpha}, \mathrm{ABC}$, and GA indices for this important class of networks [10].

Unlike the other classes of linear polymers, the nanostar dendrimers can be constructed with a well-defined molecular structure i.e. monodisperse. The dendrimers have a uniform and well-defined size and shape, which are of vital interest in the biomedical applications, pharamcology and nanotechnology. They have three structural units named as the core, branching units and the terminal end groups. The charge on end groups plays a vital role in the exploration of the dendrimers as drug delivery vehicles. Graph theory has found a considerable amount use in the area of research of nanobiotechnology in the last few decades. Cheminformatics is a new subject which is a combination of chemistry, mathematics and information science. It studies the quantitative structure activity (QSAR) and structure property (QSPR) relationships that are used to predict the biological activities and properties of chemical compounds. In chemical graph theory, a molecular graph is a representation of the structural formula of a chemical compound in terms of graph theory, whose vertices correspond to the atoms of the compound and edges correspond to chemical bonds. A graph $G$ is a pair $G=(V$;E) consisting of a finite set $V$ of vertices and $E$ is a set of pairs of elements in V. A graph $G$ is connected if there exists a connection between any pair of vertices in $G$. The degree $\operatorname{deg}(\mathrm{v})$ of $\mathrm{v}$ is the number of edges of $G$ incident with $v$. The degree $\operatorname{deg}(v)$ of $v$ is the number of edges of $G$ incident with $\mathrm{v}$.

Nano-biotechnology is a rapidly advancing area of scientific and technological opportunity that applies the tools and processes of nanofabrication to build devices for studying bio-systems. Dendrimers are one of the main objects of this new area of science. A dendrimer is an arterially manufactured or synthesized molecule built up from branched units called monomers using a nanoscale fabrication process. From a polymer chemistry point of view, dendrimers are nearly perfect monodisperse macromolecules with a regular and highly branched three dimensional architecture. They consist of three major architectural components core, branches and end groups. New branches emitting from a central core are added in steps until a tree-like structure is created.

These macromolecules and, more precisely, those containing phosphorus are used in the formation of nanotubes, micro and macrocapsules, nanolatex, colored glasses, chemical sensors, modified electrodes and so on [1,12]. Dendrimer is a synthetic 3dimensional macromolecule that is prepared in a stepwise fashion from simple branched monomer units [13].

A molecular structure descriptor or a topological index is actually a numeric quantity associated with chemical constitution purporting for correlation of chemical structure with many physiochemical properties, chemical reactivity or you can say biological activity.

A great variety of such indices are studied and used in theoretical chemistry. Among them, a large number of indices, depend only on vertex degrees of the molecular graph. A considerable amount of these vertex-degreebased topological indices can be represented as the sum of edge contributions of graph. These kind of vertex-degree-based topological indices are known as bond incident degree indices (BID) whose general form $[4,5,7,8]$ is given below.

$$
T I(G)=\sum_{1 \leq i<j \leq \square} \Theta_{i, j} \cdot x_{i, j}
$$

where $\Theta_{i, j}$ is a non-negative real valued function depending on $i$ and $j$ with $\Theta_{i, j}=\Theta_{j, i} ; \Delta$ is the maximum vertex degree in the graph $\mathrm{G}$ and $\mathrm{x}_{\mathrm{i}, \mathrm{j}}$ is the number of edges in 
the graph G connecting the vertices of degrees $i$ and $j$.

i) If $\left(\Theta_{i, j}\right)=(i \times j)^{\alpha}$ and $\alpha \in \mathrm{R},\{0\}$, then $\mathrm{TI}$ is general Randić index (variable second Zagreb index). Moreover, if $\alpha=-1 / 2,1,-1$ then TI is Randić index, second Zagreb index, modified second Zagreb index respectively.

ii) If $\left(\Theta_{i, j}\right)=(i+j)^{\beta}$ and $\beta \in \mathrm{R},\{0\}$, then TI is the general sum-connectivity index. If $\beta=-1 / 2,1$ then TI is the sum-connectivity index, first Zagreb index respectively.

iii) If $\left(\Theta_{i, j}\right)=\left(\frac{i+j-2}{i \times j}\right)^{\gamma}$ and $\gamma \in \mathrm{R},\{0\}$, then $\mathrm{TI}$ is the general atom-bond connectivity index. For $\gamma=1 / 2 ;-3$, then TI is the atom-bond connectivity index and augmented Zagreb index respectively.

iv) If $\left(\Theta_{i, j}\right)=\left(\frac{2 \sqrt{i \times j}}{i+j}\right)^{\lambda}$ and $\lambda \in \mathrm{R}^{+}$, then TI is the ordinary generalized geometric arithmetic index. For $\lambda=1$, TI is the first geometricarithmetic index.

v) If $\left(\Theta_{i, j}\right)=\frac{2}{i+j}$, then TI is the harmonic index.

vi) If $\left(\Theta_{i, j}\right)=|i-j|$, then $\mathrm{TI}$ is the Albertson index.

vii) If $\left(\Theta_{i, j}\right)=\frac{\ln i}{i}+\frac{\ln j}{j}$, then TI is logarithm of the first multiplicative.

viii) If $\left(\Theta_{i, j}\right)=\ln (i+j)$, then TI is logarithm of the second multiplicative Zagreb index.

ix) If $\left(\Theta_{i, j}\right)=\ln i+\ln j$, then TI is logarithm of the modified first multiplicative Zagreb index.

Nanotechnology is a technology that deals with small structures or small sized materials. A nanometer $(\mathrm{nm})$ is one billionth of a meter, or $10^{-9} \mathrm{~m}$. Nanostructured materials are those with at least one dimension falling in nanometer scale, and include nanoparticles (including quantum dots, when exhibiting quantum effects nanorods and nanowires, thin lms, and bulk materials made of nanoscale building blocks or consisted of nanoscale structures. Many technologies have been explored to fabricate nanostructures [6].

Diudea was the first chemist who considered the problem of computing topological indices of nanostructures $[14,15]$. Ashrafi continued this pioneering work of Diudea [2,3]. In recent years, the problem of computing topological indices of nanostructures has attracted substantial attention from many researchers $[9,11]$.

\section{Nanostar dendrimer}

Consider a graph $\mathrm{G}$ on $\mathrm{n}$ vertices, where $\mathrm{n} \geq 2$. The maximum possible vertex degree in such a graph is $n 1$. Suppose $\mathrm{x}_{\mathrm{ij}}$ denotes the number of edges of $\mathrm{G}$ connecting vertices of degrees $i$ and $\mathrm{j}$. Clearly, $\mathrm{x}_{\mathrm{ij}}=\mathrm{x}_{\mathrm{ji}}$. We now consider two infinite classes $\mathrm{NS}_{1}[\mathrm{n}]$ and $\mathrm{NS}_{2}[\mathrm{n}]$ of nanostar dendrimers Figures 1 and 2). Our aim is to compute the BID indices for two classes of these nanostar dendrimers (Figure 3). We consider the molecular graph of $\mathrm{NS}_{1}[\mathrm{n}]$ with four similar branches and three extra edges, where $\mathrm{n}$ is the steps of growth in this type of nanostars dendrimer (Figure 1).

Define $\mathrm{x}_{12}$ to be the number of edges connecting a vertex of degree 1 with a vertex of degree $2, \mathrm{x}_{13}$ to be the number of edges connecting a vertex of degree 1 with a vertex of degree $3, \mathrm{x}_{22}$ to be the number of edges connecting two vertices of degree 2 and $x_{23}$ to be the number of edges connecting a vertex of degree 2 with a vertex of degree 3 . Also $x_{i j}^{\prime}$ denotes the number of edges connecting vertices of degrees $i$ and $j$ in each branch $(\mathrm{i} ; \mathrm{j} \leq 4)$. It is obvious that that $x_{12}=4 x_{12}^{\prime}, x_{22}=4 x_{22}^{\prime}+1, x_{13}=4 x_{13}^{\prime}, x_{23}=4 x_{23}^{\prime}+2$. On the other hand, a simple calculation shows that $x_{12}^{\prime}=2^{n-1}$. Therefore $x_{12}=4 x_{12}^{\prime}=2.2^{n}$. Using a similar argument, one can see that $\mathrm{x}_{22}^{\prime}=3 \mathrm{n}-3$ then $x_{22}=12.2^{n}-11, x_{13}^{\prime}=2^{n}-1$, then $x_{13}=4 x_{13}^{\prime}=4.2^{n}-4$ and finally $x_{23}^{\prime}=3\left(2^{n}-1\right)+\left(2^{n-1}-1\right)$, so $x_{23}=4 x_{23}^{\prime}+2=14.2^{n}-14$. 


\section{Calculation of BID indices of $\mathrm{NS}_{1}[\mathrm{n}]$}

Consider the molecular graph of $\mathrm{NS}_{1}[\mathrm{n}]$ with four similar branches and three extra edges,

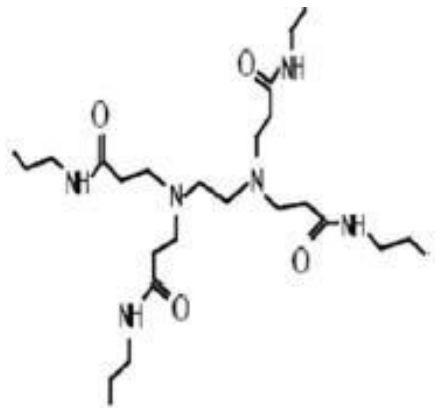

where $\mathrm{n}$ is the step of growth in this type of nanostar dendrimer as in Figure 1.

FIGURE $1 \mathrm{NS}_{1}[1]$ and $\mathrm{NS}_{1}[\mathrm{n}]$ polypropylenimine octaamin dendrimer

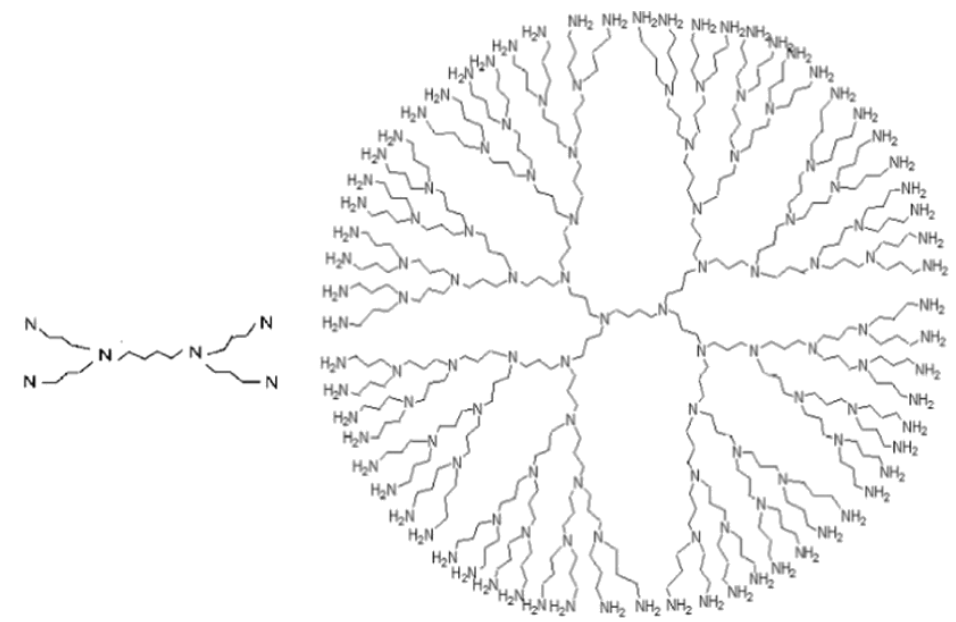

FIGURE $2 \mathrm{NS}_{2}[1]$ and $\mathrm{NS}_{2}[\mathrm{n}]$ polypropylenimine octaamin dendrimer

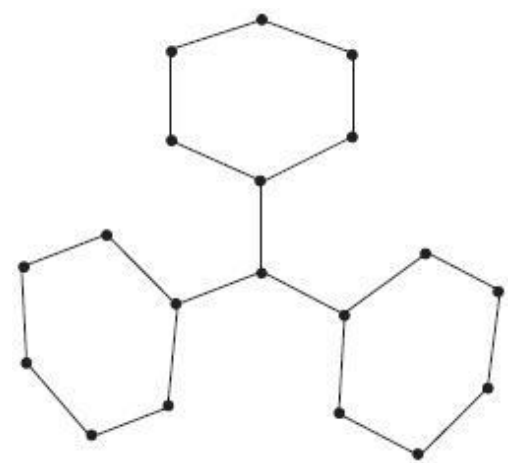

FIGURE 3 The nanostar dendrimer $D_{n}$ for $n=1$

Theorem 3.0.1. Consider the graph of

$$
\begin{aligned}
& \operatorname{TI}(G)=\Theta_{1,2} \times 2^{n+1}+\Theta_{1,3} \times 4\left(2^{n}-1\right) \\
& +\Theta_{2,2} \times\left(12.2^{n}-11\right)+\Theta_{2,3} \times\left(14.2^{n}-14\right) .
\end{aligned}
$$
bond incident degree (BID) index form is equal to:

TABLE 1 The edge partition of $G=N S_{1}[n]$ based on degrees of end vertices of each edge

\begin{tabular}{ccccc}
$\mathbf{x}_{\mathbf{i}, \mathbf{j}}$ & $(\mathbf{1 , 2 )}$ & $\mathbf{( 1 , 3 )}$ & $\mathbf{( 2 , 2 )}$ & $(\mathbf{2 , 3 )}$ \\
\hline Number of edges & $2^{\mathrm{n}+1}$ & $4\left(2^{\mathrm{n}}-1\right)$ & $12 \times 2^{\mathrm{n}}-11$ & $14 \times 2^{\mathrm{n}}-14$ \\
\hline
\end{tabular}


Proof. In order to prove (2), use Table 1 and Case $(1,2)$. It follows that

$$
\begin{aligned}
& T I(G)=\sum_{1 \leq i<j \leq \square} \Theta_{i, j} \cdot x_{i, j} \\
& T I(G)=\Theta_{1,2} \times x_{1,2}+\Theta_{1,3} \times x_{1,3}+\Theta_{2,2} \times x_{2,2}+\Theta_{2,3} \times x_{2,3} \\
& T I(G)=\Theta_{1,2} \times 2^{n+1}+\Theta_{1,3} \times 4\left(2^{n}-1\right) \\
& +\Theta_{2,2} \times\left(12.2^{n}-11\right)+\Theta_{2,3} \times\left(14.2^{n}-14\right)
\end{aligned}
$$

By using (2), we can calculate any BID index. If $\Theta_{i, j}=(i \times j)^{\alpha}$.

By using Table 2, Case $(1,3)$ and $\alpha=-1 / 2,2 ; 1$;1, we compute TI for Randić, second Zagreb and modified second Zagreb indices, respectively.

$T I(G)= \begin{cases}2^{n}\left(\frac{3 \sqrt{2}+4 \sqrt{3}+7 \sqrt{6}+18}{3}\right)-\left(\frac{8 \sqrt{3}+14 \sqrt{6}+33}{6}\right) & \text { if } \alpha=-1 / 2, \\ 3\left(2^{n+4}\right)+2^{n+2}+96\left(2^{n}\right)-140 & \text { if } \alpha=1, \\ \left(\frac{2^{n+2}+19\left(2^{n}\right)}{3}\right)-\frac{77}{12} & \text { if } \alpha=-1\end{cases}$

If $\Theta_{i, j}=(i+j)^{\beta}$.

TABLE 2 The numbersof non-negative real valued function depending $\mathrm{x}_{\mathrm{i}, \mathrm{j}}$ in cases $\alpha, \beta$

\begin{tabular}{ccccc}
\hline $\mathbf{x}_{\mathbf{i}, \mathbf{j}}$ & $(\mathbf{1 , 2})$ & $\mathbf{( 1 , 3 )}$ & $\mathbf{( 2 , 2 )}$ & $\mathbf{( 2 , 3 )}$ \\
$\boldsymbol{\alpha}=-\mathbf{1} / \mathbf{2}$ & $\sqrt{2} / 2$ & $\sqrt{3} / 3$ & $1 / 2$ & $\sqrt{6} / 6$ \\
$\boldsymbol{\alpha}=\mathbf{1}$ & 2 & 3 & 4 & 6 \\
$\boldsymbol{\alpha}=-\mathbf{1}$ & $1 / 2$ & $1 / 3$ & $1 / 4$ & $1 / 6$ \\
$\boldsymbol{\beta}=-\mathbf{1} / \mathbf{2}$ & $\sqrt{3} / 3$ & $1 / 2$ & $1 / 2$ & $\sqrt{5} / 5$ \\
$\boldsymbol{\beta}=\mathbf{1}$ & 3 & 4 & 4 & 5 \\
$\boldsymbol{\gamma}=\mathbf{1} / \mathbf{2}$ & $\sqrt{2} / 2$ & $\sqrt{6} / 3$ & $\sqrt{2} / 2$ & $\sqrt{2} / 2$ \\
$\boldsymbol{\gamma}=-\mathbf{3}$ & 8 & $27 / 8$ & 8 & 8 \\
$\boldsymbol{\lambda}=\mathbf{1}$ & $2 \sqrt{2} / 3$ & $\sqrt{3} / 2$ & 1 & $2 \sqrt{6} / 5$ \\
\hline
\end{tabular}

By using Table 2 , Case $(1,3)$ and $\beta=-1 / 2 ; 1$, we compute TI for sum connectivity and first Zagreb indices, respectively.

$T I(G)= \begin{cases}2^{n+1}\left(\frac{9+\sqrt{3}}{3}\right)+\left(2^{n}-1\right)\left(\frac{10+14 \sqrt{5}}{5}\right)-\frac{11}{2} & \text { if } \beta=-1 / 2, \\ 3.2^{n+4}+3.2^{n+1}+86.2^{n}-130 & \text { if } \beta=1,\end{cases}$

If $\Theta_{i, j}=\ln i+\ln j$.

By using Table 3 and Case $(1,3)$, we compute TI for logarithm of modified first multiplicative Zagreb index.
$T I(G)=\ln 2\left(2^{n+4}+3.2^{n+3}\right)+\ln 3.2^{n+2}$

$+7 . \ln 3.2^{n+1}-(36 \cdot \ln 2+18 . \ln 3)$

If $\Theta_{i, j}=\left(\frac{i+j-2}{i \times j}\right)^{\gamma}$.

TABLE 3 The value of $\Theta_{i, j}$ on based the edge partition $\mathrm{x}_{\mathrm{i}, \mathrm{j}}$

\begin{tabular}{ccccc}
\hline $\mathbf{x}_{\mathbf{i}, \mathbf{j}}$ & $(\mathbf{1 , 2})$ & $\mathbf{( 1 , 3 )}$ & $\mathbf{( 2 , 2 )}$ & $\mathbf{( 2 , 3 )}$ \\
\hline $\boldsymbol{\Theta}_{\mathbf{i}, \mathbf{j}}$ & $\ln (2)$ & $\ln (3)$ & $\ln (4)$ & $\ln (2)+\ln (3)$ \\
\hline
\end{tabular}

By using Table 2, Case $(1,3)$ and $\gamma=1 / 2 ;-3$, we compute TI for atom bond connectivity and augmented Zagreb indices, respectively.

$T I(G)= \begin{cases}2^{n+7 / 2}+\left(\frac{2^{n+5 / 3} \sqrt{3}}{3}\right)+3.2^{n+3 / 2}-\left(\frac{75 \sqrt{2}+8 \sqrt{6}}{6}\right) & \text { if } \gamma=1 / 2, \\ 3.2^{n+5}+2^{n+4}+251.2^{n-1}-\frac{427}{2} & \text { if } \gamma=-3,\end{cases}$

If $\Theta_{i, j}=\left(\frac{2 \sqrt{i \times j}}{i+j}\right)^{\lambda}$.

By using Table 2 and Case $(1,3)$, we compute TI for first geometric-arithmetic index.

$T I(G)=\frac{2^{n+5 / 2}}{3}+3.2^{n+2}+\left(2^{n}-1\right)\left(\frac{10 \sqrt{3}+28 \sqrt{6}}{5}\right)-11$

If $\Theta_{i, j}=\frac{2}{i+j}$.

By using Table 4 and Case $(1,3)$, we compute TI for Harmonic index.

$T I(G)=\frac{2^{n+2}}{3}+\frac{34\left(2^{n+1}\right)}{5}-\frac{131}{10}$. If $\Theta_{i, j}=\frac{\ln i}{i}+\frac{\ln j}{j}$.

TABLE 4 The value of $\Theta_{i, j}$ on based the edge partition $\mathrm{x}_{\mathrm{i}, \mathrm{j}}$

\begin{tabular}{ccccc}
\hline $\mathbf{x}_{\mathbf{i}, \mathbf{j}}$ & $(\mathbf{1 , 2 )}$ & $\mathbf{( 1 , 3 )}$ & $\mathbf{( 2 , 2 )}$ & $\mathbf{( 2 , 3 )}$ \\
\hline $\boldsymbol{\Theta}_{\mathbf{i}, \mathbf{j}}$ & $2 / 3$ & $1 / 2$ & $1 / 2$ & 0.2 \\
$\boldsymbol{\Theta}_{\mathbf{i}, \mathbf{j}}$ & $\ln (\sqrt{2})$ & $\ln (3)^{1 / 3}$ & $\ln (2)$ & $\ln (\sqrt{2})+\ln (3)^{1 / 3}$ \\
$\boldsymbol{\Theta}_{\mathbf{i}, \mathbf{j}}$ & 1 & 2 & 0 & 1 \\
\hline
\end{tabular}

By using Table 4 and Case $(1,3)$, we compute TI for logarithm of first multiplicative Zagreb index, If $\Theta_{i, j}=|i-j|$.

$$
T I(G)=2^{n}(\ln (764411904))-\ln (191102976)
$$

By using Table 4 in equation (2), we compute TI for Albertson index.

$$
T I(G)=2^{n+1}+22\left(2^{n}-1\right) .
$$


If $\Theta_{i, j}=\ln (i+j)$, then we have

$\Theta_{1,2}=\ln 3, \Theta_{1,3}=\ln 4, \Theta_{2,2}=\ln 4$ and $\Theta_{2,3}=\ln 5$.

$T I(G)=\ln 3 \cdot 2^{n+1}+(4 \cdot \ln 4+14 \ln 5) \cdot 2^{n-1}$

$+12 \cdot \ln 4.2^{n}-11 \cdot \ln 4$

\section{Computation of BID indices for $\mathrm{NS}_{2}[\mathrm{n}]$}

We now consider the second class of nanostar dendrimers $\mathrm{NS}_{2}[\mathrm{n}]$, where $\mathrm{n}$ is the steps of growth. Since the molecular graph of $\mathrm{G}$ has four similar branches and five extra edges as in Figure 2, we have $x_{12}=4 x_{12}^{\prime}, x_{22}=4 x^{\prime}{ }_{22}+3$, and $x_{23}=4 x_{23}^{\prime}+2$. After simple calculation, we have $x_{12}^{\prime}=2^{n-1}, x^{\prime}{ }_{22}=2\left(2^{n}-1\right), x_{23}{ }_{23}=3.2^{n-1}-2$. It is easy to compute $x_{12}=2^{n+1}, x_{22}=8.2^{n}-5$ and $x_{23}=6.2^{n}-6$.

Theorem 4.0.1. Consider the graph of $\mathrm{G}=\mathrm{NS}_{2}[\mathrm{n}]$ nanostar dendrimer, then its bond incident degree (BID) index is equal to:

$T I(G)=\Theta_{1,2} \times 2^{n+1}+\Theta_{2,2} \times\left(8.2^{n}-5\right)+\Theta_{2,3} \times\left(6.2^{n}-6\right)$.

TABLE 5 The edge partition of $\mathrm{G}=\mathrm{NS}_{2}[\mathrm{n}]$ based on degrees of end vertices of each edge

\begin{tabular}{ccrr}
\hline $\mathbf{x}_{i, j}$ & $(1,2)$ & $(2,2)$ & $(2,3)$ \\
\hline $\begin{array}{c}\text { Number of } \\
\text { edges }\end{array}$ & $2^{n+1}$ & $8 \times 2^{\mathrm{n}-5}$ & $6 \times 2^{\mathrm{n}-6}$ \\
\hline
\end{tabular}

Proof. In order to prove (3), use Table 5 and Case $(1,2)$. It follows that

$T I(G)=\sum_{1 \leq i<j \leq} \Theta_{i, j} \cdot x_{i, j}$

$T I(G)=\Theta_{1,2} \times x_{1,2}+\Theta_{2,2} \times x_{2,2}+\Theta_{2,3} \times x_{2,3}$.

After simplification and rearranging the terms, we get

$T I(G)=\Theta_{1,2} \times 2^{n+1}+\Theta_{2,2} \times\left(8.2^{n}-5\right)+\Theta_{2,3} \times\left(6.2^{n}-6\right)$

Now using (3), we can calculate any BID index. $\Theta_{i, j}=(i \times j)^{\alpha}$.

By using Table 6 and Case $(2,3)$, we compute Randić, second Zagreb and modified second Zagreb indices when $\alpha=-1 / 2,2 ; 1 ;-1$, respectively.

$T I(G)= \begin{cases}2^{n+2}+2^{n+1 / 2}+\sqrt{6} \cdot 2^{n}-\left(\frac{5+2 \sqrt{6}}{2}\right) & \text { if } \alpha=-1 / 2, \\ 2^{n+5}+2^{n+4}-56 & \text { if } \alpha=1, \\ 2^{n+2}-\frac{9}{4} & \text { if } \alpha=-1\end{cases}$

If $\Theta_{i, j}=(i+j)^{\beta}$.

TABLE 6 The numbersof non-negative real valued function depending $\Theta_{i, j}$ in cases $\alpha, \beta$

\begin{tabular}{cccc}
\hline $\boldsymbol{\Theta}_{\mathrm{i}, \mathrm{j}}$ & $(\mathbf{1 , 2 )}$ & $\mathbf{( 2 , 2 )}$ & $\mathbf{( 2 , 3 )}$ \\
$\boldsymbol{\alpha}=-\mathbf{1} / \mathbf{2}$ & $\sqrt{2} / 2$ & $1 / 2$ & $\sqrt{6} / 6$ \\
$\boldsymbol{\alpha}=\mathbf{1}$ & 2 & 4 & 6 \\
$\boldsymbol{\alpha}=-\mathbf{1}$ & $1 / 2$ & $1 / 4$ & $1 / 6$ \\
& $\sqrt{3} / 3$ & $1 / 2$ & $\sqrt{5} / 5$ \\
$\boldsymbol{\beta}=-\mathbf{1} / \mathbf{2}$ & $\sqrt{3}$ & 4 & 5 \\
$\boldsymbol{\beta}=\mathbf{1}$ & 3 & $\sqrt{2} / 2$ & $\sqrt{2} / 2$ \\
$\gamma=1 / 2$ & $\sqrt{2} / 2$ & $\sqrt{2}$ & 8 \\
$\boldsymbol{\gamma}=-\mathbf{3}$ & 8 & 8 & $2 \sqrt{6} / 5$ \\
$\boldsymbol{\lambda}=\mathbf{1}$ & $2 \sqrt{2} / 3$ & 1 & \\
\hline
\end{tabular}

By using Table 6 and Case $(2,3)$, we compute TI for sum connectivity and first Zagreb indices for $\beta=-1 / 2 ; 1$, respectively.

$T I(G)= \begin{cases}2^{n+2}+2^{n+1}\left(\frac{9 \sqrt{5}+5 \sqrt{3}}{15}\right)-\left(\frac{25+12 \sqrt{5}}{10}\right) & \text { if } \beta=-1 / 2, \\ 2^{n+5}+9.2^{n+2}-50 & \text { if } \beta=1,\end{cases}$

If $\Theta_{i, j}=\left(\frac{i+j-2}{i \times j}\right)^{\gamma}$.

By using the Table 6 in Case $(2,3)$, we compute TI for atom bond connectivity index and augmented Zagreb index for $\gamma=1 / 2 ;-3$, respectively.

$T I(G)= \begin{cases}2^{n+7 / 2}-\frac{11}{\sqrt{2}} & \text { if } \gamma=1 / 2, \\ 2^{n+7}-88 & \text { if } \gamma=-3,\end{cases}$

If $\Theta_{i, j}=\left(\frac{2 \sqrt{i \times j}}{i+j}\right)^{\lambda}$.

By using Table 6 and Case $(2,3)$, we compute TI for first geometric-arithmetic index.

$T I(G)=2^{n+5 / 2}\left(\frac{5+9 \sqrt{3}}{15}\right)+2^{n+3}-\left(\frac{25+12 \sqrt{6}}{5}\right)$ 
If $\Theta_{i, j}=\frac{2}{i+j}$.

By using Table 6 and Case $(2,3)$, we compute TI for harmonic index.

$$
\text { If } \Theta_{i, j}=|i-j| . T I(G)=\frac{29.2^{n+2}}{15}-\frac{49}{10}
$$

By using Table 7 and Case $(2,3)$, we compute TI for Albertson index.

$$
\text { If } \Theta_{i, j}=\frac{\ln i}{i}+\frac{\ln j}{j} . T I(G)=2^{n+3}-6
$$

TABLE 7 The value of $\Theta_{i, j}$ on based the edge partition $\mathrm{x}_{\mathrm{i}, \mathrm{j}}$ in cases $\lambda$ and $\gamma$

\begin{tabular}{cccc}
\hline $\mathbf{x}_{\mathbf{i}, \mathbf{j}}$ & $(\mathbf{1 , 2 )}$ & $\mathbf{( 2 , 2 )}$ & $\mathbf{( 2 , 3 )}$ \\
\hline$\Theta_{\mathrm{i}, \mathrm{j}}$ & 1 & 0 & 1 \\
$\Theta_{\mathrm{i}, \mathrm{j}}$ & $2 / 3$ & $1 / 2$ & $2 / 5$ \\
$\Theta_{\mathrm{i}, \mathrm{j}}$ & $\ln (\sqrt{2})$ & $\ln (2)$ & $\ln (\sqrt{2})+\ln (3)^{1 / 3}$ \\
\hline
\end{tabular}

By using Table 7 and Case $(2,3)$, we compute TI for logarithm of first multiplicative Zagreb index.

$T I(G)=\ln 2\left(2^{n+3}+2^{n+2}\right)+\ln 3 \cdot 2^{n+1}-(8 \cdot \ln 2+2 \cdot \ln 3)$ If $\Theta_{i, j}=\ln (i+j)$.

By using Table 7 and Case $(2,3)$, we compute TI for logarithm of second multiplicative Zagreb index.

$T I(G)=\ln 2.2^{n+4}+2^{n+1}(\ln 3+3 \cdot \ln 5)-(10 \cdot \ln 2+6 \cdot \ln 5)$

If $\Theta_{i, j}=\ln i+\ln j$, then we have $\Theta_{1,2}=\ln 2$, $\Theta_{2,2}=\ln 4, \Theta_{2,3}=\ln 2+\ln 3$.

TABLE 8 The $\ln (i+j)$ of edge $x_{i, j}$

\begin{tabular}{cccc}
$\mathbf{x}_{\mathbf{i}, \mathbf{j}}$ & $(\mathbf{1}, \mathbf{2})$ & $\mathbf{( 2 , 2 )}$ & $\mathbf{( 2 , 3 )}$ \\
\hline $\ln (i+j)$ & $\ln (3)$ & $\ln (4)$ & $\ln (5)$ \\
\hline
\end{tabular}

Using above values in Case $(2,3)$, it follows that $T I(G)=\ln 2\left(2^{n+4}+2^{n+3}\right)$ $+3 \cdot \ln 3 \cdot 2^{n+1}-(16 \cdot \ln 2+6 \cdot \ln 3)$.

\section{Conclusion}

In this paper, we deal with nanostar dendrimers $\mathrm{NS}_{1}[\mathrm{n}]$ and $\mathrm{NS}_{2}[\mathrm{n}]$ to establish a general expression for calculating the bond incident degree indices. The generalized form of BID indices is then used to compute several well-known BID indices. The computed results in this paper will be quite helpful to study the physiochemical properties, chemical reactivity or biological activities. In future, we are interested to study and design some new network architectures in order to study their bond incident degree indices and then study their physical and chemical properties like boiling point, stability, strain energy etc.

\section{Acknowledgments}

This research is partially supported by School of Mathematics and Physics, Anhui Jianzhu University, Hefei 230601, China and COMSATS Attock and National University of Sciences and Technology, Islamabad, Pakistan and also this research is supported by the Start-up Research Grant 2016 of United Arab Emirates University (UAEU), Al Ain, United Arab Emirates via Grant No. G00002233 and UPAR Grant of UAEU via Grant No. G00002590.

\section{Orcid:}

Jia-Bao Liu: https://orcid.org/0000-00029620-7692

Abdul Qudair Baig: https://orcid.org/00000002-8913-5221

waqas Khalid: https://orcid.org/0000-00015527-6563

Mohammad Reza Farahani:

https://orcid.org/0000-0003-2969-4280

\section{References}

[1] A.R. Ashrafi, M. Mirzargar, Indian J. Chem., 2008, 47, 538-541.

[2] A.R. Ashrafi, F. Rezaei, MATCH Commun. Math. Comput. Chem., 2007, 57, 243-250.

[3] A.R. Ashrafi, A. Loghman, MATCH Commun. Math. Comput. Chem., 2006, 55, 447-452.

[4] B. Hollas, MATCH Commun. Math. Comput. Chem., 2005, 54, 177-187.

[5] D. Vukičević, M. Gašperov, Croat. Chem. Acta., 2010, 83, 243-260. 
[6] G. Cao, Y. Wang, Nanostructures \& Nanomaterials, Synthesis, Properties, Applications. World Scientific Series in Nanoscience and Nanotechnology, 2nd Edition, 2, 2011.

[7] I. Gutman, Croat. Chem. Acta., 2013, 86, 351-361.

[8] J. Rada, R. Cruz, I. Gutman, Chemical Physics Letters, 2013, 572, 154-157.

[9] L. Yang, H. Hua, Optoelectron. Adv. Mat., 2012, 6, 660-663.

[10] M. Baca, J. Horvathova, M. Mokrisova, A. Semanicova-Fenovckova, A. Suhanyiova, Can. J. Chem., 2015, 93, 1157-1160.

[11] M. Ghorbani, H. Mesgarani, S. Shakeraneh, Optoelectron. Adv. Mat., 2011, 5, $324-326$.

[12] M.V. Diudea, A.E. Vizitiu, M. Mirzagar, A.R. Ashrafi, Carpathian J. Math., 2010, 26, 59-66. [13] M.V. Diudea, G. Katona, Advan. Dendritic Macromol., 1999, 4, 135-201.
[14] M.V. Diudea, M. Stefu, B. Parv, P.E. John, Croat. Chem. Acta., 2004, 77, 111-115.

[15] P.E. John, M. V. Diudea, Croat. Chem. Acta., 2004, 77, 127-132.

[16] W. Gao, M.R. Farahani, L. Shi, Acta Medica Mediterr, 2016, 32, 579-585.

[17] W. Gao, W.F. Wang, Comput. Math. Method. M., 2015, Article ID 418106, 10 pages, doi:10.1155/2015/418106

[18] W. Gao, W.F. Wang, M.R. Farahani, J. Chem-NY, 2016, Article ID 3216327, 8 pages, https://doi.org/10.1155/2016/3216327.

How to cite this article: Jia-Bao Liu, Abdul Qudair Baig, Muhammad Imran*, Waqas Khalid, Muhammad Saeed, Mohammad Reza Farahani. Computation of bond incident degree (BID) indices of complex structures in drugs. Eurasian Chemical Communications, 2020, 2(6), 672-679. Link: http://www.echemcom.com/article_105 097.html 